\title{
NEUE ANSICHTEN ÜBER DIE MORPHOLOGIE DES FLOH- KOPFES, SOWIE ÜBER DIE ONTOGENIE, PHYLOGENIE UND SYSTEMATIK DER FLÖHE.
}

\author{
VON DR. A. C. OUdemans (Arnhem, Holland).
}

\author{
(Tafel XII., XIII.)
}

\section{Einleitung.}

A LS ich in der Tijdschrift voor Entomologie (vol. li., p. 89, 1908) schrieb, dass 1 einige Flöhe einen in zwei Teile gegliederten Kopf hätten, dachte ich wenig daran, dass diese und weitere Entdeckungen auf diesem Gebiete solche weitgehende Folgen haben würden.

Die Tatsache allein, dass ein Insekt einen gegliederten Kopf besitzt, ist eine so ausserordentliche, dass es sonderbar genannt werden muss, dass sie vor mir niemand wahrgenommen hat. Vergebens suchte ich danach in der Literatur. Ich finde nur einen Passus, welcher annäherend anf diese Kopfgliederung hinweist; die Autoren haben sie aber offenbar nicht als solche erkannt.

Vor einigen Wochen nämlich fand ich zufällig den folgenden Satz in Jordan und Rothschild's "Revision of the Sarcopsyllidae" (Thompson, Yates \& Johnston Laboratories Report vii. p. 19, 1906):

"The head of the Siphonaptera is divided by the antennal groove into an anterior or frontal portion and a posterior or occipital portion. The antennal groove is usually prolonged dorsally as a narrow slit in the $\delta$ of Siphonaptera, the grooves of the two sides of the head often meeting on the top. There is normally also an internal incrassation of the skeleton from the groove upwards in both sexes. This arrangement is well marked in the Sarcopsyllid genus Echidnophaga. In the genera Hectopsylla and Dermatophilus the dorsal prolongation of the antennal groove and the internal thickening of the chitin are absent in both sexes, the genera Dermatophilus and Hectopsylla being in this respect more specialised than Echidnophaga. Such a specialisation is not confined to these two genera of Sarcopsyllidae. We meet with it also among the Pulicidae in the genera Chaetopsylla and Vermipsylla."

Jordan und Rothschild haben also zuerst wahrgenommen und publiciert-

1. Dass bei vielen Männchen der Suctoria die Antennengruben sich dorsalwärts als enge Spalten verlängern, so dass die Gruben der beiden Kopfseiten sich am Scheitel begegnen;

2. Dass bei vielen Flöhen, und dann in beiden Geschlechtern, sich eine inwendige Verdickung des Skelettes von den Antennengruben anfwärts befindet (Echidnophaga und die meisten Pulicidae);

3. Dass bei anderen Flöhen die oben erwähnten spaltförmigen Fortsetzungen der Antennengruben in beiden Geschlechtern fehlen (Hectopsylla, Dermatophilus, Chaetopsylla, und Vermipsylla). 
Unabhängig von den genannten Antoren bemerkte ich im Anfang des vorigen Jahres (1908) dieselbe Chitinverdicknng bei einigen Flohgattungen und das Fehlen derselben bei anderen. Andere Tatsachen wurden dagegen zuerst von mir wahrgenommen, und ich publicierte sie in der genannten Tijdschrift.

Es scheint mir notwendig zu sein hier kurz zu wiederholen, was ich dort gesagt habe.

"Die meisten Flöhe besitzen einen gewöhnlichen ungegliederten Kopf (caput integrum). Es giebt aber Flöhe mit einem buchstäblich in zwei Teile gegliederten Kopfe, nämlich in einen Vorderkopf (pars anterior) und einen Hinterkopf (pars posterior). Hierzn gehören nur die Ischnopsyllidae. Die Teile sind selbst beweglich miteinander verbunden. Die Beweglichkeit ist nicht gross nnd besteht darin, dass sie nicht nur ein wenig seitwärts, sondern anch ein wenig anf nnd nieder geschehen kann, und zwar um einen Gelenkhöcker (condylus), welcher sich ungefähr im Zentrnm des Kopfes befindet und stark chitinisirt ist. Die Folge der ventralen Flexion ist, dass der freie Hinterrand (collare) der pars anterior sich von der pars posterior abhebt (Fig. 3), und dass der dorsale Teil der Antennengrube sich erweitert, während nmgekehrt der ventrale Teil derselben sich verengt. Im entgegengesetzen Falle schliesst sich das collare genan an die pars posterior an, verengt sich der dorsale Teil der Antennengrube, und erweitert sich der ventrale Teil derselben. Solch einen Kopf nenne ich ein caput fractum.

"Die pars anterior eines solchen gegliederten Kopfes besitzt, wie die pars posterior, die Thorax-Segmente und die Abdominal-Tergite, einen breiten, platten Hinterrand oder Kragen (collare), welcher über der pars posterior liegt, wïhrend diese wie alle hinter ihr folgenden Thoracal- und Abdominal-Tergite vorn ein Stosskissen (nodulus) besitzt, welches in eine Pfanne (fossa noduli) der pars anterior. passt.

"Die pars anterior gleicht nun einem echten Kopfe; die pars posterior ist zum Verwechseln einem Pronotum ähnlich.

"Was noch sonderbarer ist : an der pars anterior befinden sich die rudimentären Augen und die Mundwerkzenge, während an der pars posterior die Antennen befestigt sind.

"Was nun die Köpfe der Integricipita betrifft, so zeigen anch diese manche Eigentümlichkeiten. Bei allen Suctoria treffen wir jenen centralen, stark chitiuisirten Höcker (tuber centrale) mehr oder weniger entwickelt an; die Bedentung dieses Höckers war nns bei einem caput fractum klar, ist nns aber bei einem caput integrum nnverständlich.

"Bei vielen Flöhen nehmen wir noch eine andere inwendige Chitinisirung wahr. In der Kopfhöhle befindet sich nämlich, dem Kopfdache anliegend, eine sichelförmige Chitinisirnng $(f a l x)$, welche sich von der einen Antennengrube nach der anderen hienzieht. Wozn diese falx dient, ist mir nicht dentlich ; gewiss nicht zur Anheftung von Muskeln, denn diese sind am Kopfdache selbst, sowohl vor wie hinter der falx befestigt."

In der Tidjschrift (l.c.) sind noch andere Punkte erwähnt, welche ich übergehe, da sie bei der gegenwärtigen Besprechung nicht in Betracht kommen.

Als ich obenerwähnte Entdeckungen (das Vorhandensein eines tuber centrale, das Fehlen oder Vorhandensein einer falx und der Kopfgliederung) publicierte, hatte ich nur die folgenden Floharten verglichen:

Ceratophyllus sciurorum (Schrank) nnd fasciatus (Bose), Ctenocephalus canis Curtis) und felis (Bouché), Archaeopsylla erinacei (Bouché), Pulex irritans L., 
Chaetopsylla globiceps (Tasch.), Echidnophaga gallinaceus (Westw.), Ischnopsyllus elongatus (Curtis), hexactenus (Kolen.), simplex (Rothsch.), und Nycteridopsylla pentactenus (Kolen.).

Ich teilte die Flöhe ein in Integricipita (mit den ersten sechs Gattungen) und Fracticipita (mit den letzten zwei), und war der Meinung, die Integricipita seien primitiver als die anderen.

Aber da fand ich vergangenen November anf einer Mans einige Ctenoplithalmus segnis (Schönherr). Zn meinem Erstaunen hatte diese Art auch ein caput fractum, während ich (siehe oben) der Ansicht war, dass nur die anf Fledermänsen schmarotzenden Ischnopsyllidae einen solchen hätten. Diese nene Entdecknng ïberraschte mich so, dass ich den Hon. N. Charles Rothschild, Prof. Dr. K. M. F. Kraepelin, Prof. Dr. Otto Taschenberg, Dr. G. Enderlein, Prof. Dr. Jul. Wagner und andere sofort bat, mir Weibchen der Typen oder Cotypen aller mir unbekannten Genera zur Untersuchung zuznsenden. Meine Absicht dabei war zu entscheiden, welche Genera zn den Fracticipita und welche zu den Integricipita gehörten, was ich ans den Beschreibungen und Abbildungen der verschiedenen Antoren unmöglich erkennen konnte.

Inzwischen fand ich bei meinen eigenen Exemplaren von Ctenocephalus canis (Curtis) und Ct. felis (Bouché) die Spur einer Naht, welche über die inwendige falx läuft. Diese Entdeckung war für mich eine ganz besondere, denn nun waren mir der tuber centrale und die falx nicht mehr unerklärlich. Ja noch mehr, die Fracticipita scheinen mir jetzt die primitivsten unter den jetzt lebenden Flöhen zu sein, die Integricipita dagegen leicht von den erstgenannten abzuleiten.

Die obengennanten Herren beantworteten meine Bitte mit der Znsendnng von Weibchen aller Gattungstypen, welche sie besassen, speziell stellte mir the Hon. N. C. Rothschild reiches Material zur Verfügung, nnd ich kann diesen hervorragenden Flohforschern nicht dankbar genng dafür sein.

Von den folgenden Genera konnte ich die Typen nicht untersuchen :

Anomiopsyllus Baker 1904 (Type : mudatus Baker) ; ist nach Baker mit Typhlopsylla (jetzt Spalacopsylla Oudemans 1906) verwandt. Ich werde die Gattung anch als so betrachten, obwohl man mit sogenannter "Verwandtschaft" vorsichtig sein muss.

Dasypsyllus Baker 1905 (Type : perpinnatus Baker) ist ein sonderbares Tier, das vielleicht gar nicht mit Ceratoplyllus verwandt ist, wie von Baker angenommen wird. Ich werde es trotzdem in die Nähe von Ceratophyllus setzen.

Hoplopsyllus Baker 1905 (Type : anomalus Baker). Ich konnte H. glacialis untersuchen, zweifle aber sehr an der Verwandtschaft dieser Arten, denn die abgebildete Antenne von anomalus ist zn verschieden.

Odontopsyllus Baker 1905 (Type: multispinosus Baker) ist vermutlich sehr entfernt mit Ceratoplyyllus verwandt; vorlanfig setze ich die Gattung in die Nähe der letztern.

Rhopalopsyllus Baker 1905 (Type : lutzi Baker). Ich konnte nur cleophontis Rothsch. untersuchen, welche von Rothschild jedoch als äusserst nahe verwandt mit lut zi betrachtet wird.

Stephanocircus Skuse 1890 (Type: dasyuri Skuse). Ich hatte nur Gelegenbeit S. simsoni Rothsch. zu untersuchen. Die Stephanocircus-Arten sind aber so nahe miteinauder verwandt, dass es mir wohl nicht übelgedentet werden kann, wenn ich handle als hätte ich die typische Art selbst gesehen.

Vermipsylla Schimk. (Type : alacurt Schimk.) ist mir nur ans den exacten 
Beschreibungen und Abbildnngen Wagners bekannt. Ausserdem kenne ich die Verm. hyaenae Kolen., so dass ich gewiss nicht fehlgreife, wenn ich sie in die Verwandtschaft von Chaetopsylla Kohant 1903 bringe, woranf anch nenerdings Dampf hinweist (Schrift. Physilk. Oekon. Ges. Königsb. i. Pr., vol. 49, p. 291).

Das Resultat meiner Untersuchungen lege ich nun in den folgenden Zeilen nieder :-

\section{Morphologischer Teil.}

Den sonderbarsten Kopf hat wohl Macropsylla hercules Rothsch. (Taf. XII, Fig. 1). Er ist nicht allein ein caput fractum, weil er aus zwei beweglichen Teilen besteht, sondern zeigt in der pars posterior eine schwere falx posterior. Diese erstreckt sich von der einen Kopfseite, dem Schädeldache angedrückt, nach der anderen und ist in der Mitte gewaltig dick. In der Figur ist nur dieses grosse tuber postverticale gezeichnet (= optischer Durchschnitt durch den dicksten Teil der falx posterior). Es scheint aus einer Verwachsung von zwei Lappen einer tiefen Falte der Kopfdecke entstanden zu sein. Der Raum der Falte ist mit einer hellen Chitinmasse völlig ausgefüllt. Scheinbar unter der falx, in Wirklichkeit aber der linken und rechten Kopfwand anliegend, setzen sich die beiden Hörner der falx fort (in der Figur sind nor die Grenzen mit Strichelchen angegeben). Zwischen diesen beiden Sichelhörnern liegt ein Teil des Gehirns und verläuft der Oesophagus. Weiter nach vorn sieht man die ebenfalls tiefe Kluft zwischen den zwei beweglichen Kopfteilen, das starke Stosskissen (nodulus) der pars posterior, und die damit übereinstimmende Pfanne (fossa noduli) der pars anterior, beide stark chitinisiert. Die Kluft am Vertex ist tief, reicht aber vertikal nicht weiter als die Stelle, wo der vorderste Teil des Stosskissens sich befindet und setzt sich an den Seiten des Kopfes bis an die Antennengrube fort. Das breite collare deckt einen Teill der Antennengrube.

Einen zweiten Kopftypus besitzt Stephanocircus simsoni Rothsch. (Taf. XII, Fig. 2). (Ich konnte den Typus der Gattung, dasyuri Skuse, nicht untersuchen.) Anch hier befindet sich in der Mitte der pars posterior eine schwere falx posterior mit gewaltigem tuber postverticale, worüber eine dentlich sichtbare Naht verlänft. Die beiden Hörner der falx liegen der linken und rechten Kopfwand an und sind nicht in der Figur angegeben. Zwischen ihnen liegt ein Teil des Gehirns und verläuft der Oesophagns. Am tuber postverticale kann man nicht mit Sicherheit sehen, ob er durch die Ausfüllnng einer Falte der Kopfdecke entstanden ist; wahrscheinlich ist dies aber der Fall.

Weiter nach vorn sieht man die dentliche, schmale, nicht tiefe Kluft zwischen den zwei beweglichen Kopfteilen, das starke Stosskissen (nodulus) der pars posterior. und die darauf passende Pfanne (fossa noduli) der pars anterior, beide stark chitinisiert. Die verticale Klnft reicht nicht tiefer als die Stelle, wo der vorderste Teil des Stosskissens sich befindet, setzt sich aber an den Seiten des Kopfes bis an die Antennengrube fort. Die Fortsetzung des schmalen collare deckt zum Teil die Antennengrube.

Am Kopfe der Ischnopsyllus elongatus (Cartis) (abgebildet ist I. hexactenus, weil meine Exemplare von I. elongatus nicht so gnt das collare sehen lassen, Taf. XII, Fig. 3) und an den folgenden zu beschreibenden Köpfen ist keine Spur einer falx posterior zn bemerken. Die Gliederung zwischen den beiden Kopfteilen ist dagegen sehr dentlich. Die verticale Klnft is schief nach vorne gerichtet, reicht aber nicht tiefer als die Stelle, wo sich der vorderste Teil des nodulus der pars 
posterior befindet. An den Seiten des Kopfes setzt sich jedoch die Kluft bis an die Antennengrube fort. Da die Kluft so schief nach vorn verlänft, hat sich hier am Hinterrande der pars anterior ein breiter Kragen (collare) gebildet, welcher über die pars posterior greift. Der Kragen bedeckt anch die Vorderseite der proximalen Hälfte der Antennengrube. Die Ähnlichkeit der pars posterior mit einem Pronotum ist überraschend. Vor der Antennengrube sieht man den Gelenkhöcker oder das tuber centrale. Über diesem und unter der verticalen Klnft verlänft der Oesophagus und zwar zwischen den Antennengruben und anch zwischen den chitinisierten Rändern der Hinterseite der pars anterior, und ebenfalls zwischen den chitinisierten Rändern der Vorderseite der pars posterior. Sehr weit nach vorn, gerade vor der Vorderspitze des Kopfes, bemerkt man ein dreieckiges Anhängselchen, einen mucro.

Am Kopfe von Ctenoplethalmus segnis (Schönh.) (Taf. XII, Fig. 4) ist die Gliederung der beiden Kopfteile noch dentlich, obwohl die verticale Kluft nicht tief ist; sie reicht nicht weiter als die Stelle, wo sich der nodulus befindet. Das collare ist auch dentlich und bedeckt, obwohl schmal, noch dem vorderen Teil der Antennengrube. Die Kluft setzt sich bis an die Antennengrube fort. Die Ränder der hinteren Seite der pars anterior und der vorderen Seite der pars posterior sind gnt chitinisiert. Zwischen diesen Rändern verlänft der Oesophagns (nicht mitgezeichnet). Das tuber centrale (in dieser Art hinter der Antennengrube) ist sehr dentlich.

Der Kopf der Palaeopsylla grasilis (Tasch.) (Taf. XII, Fig. 5) zeigt uns eine noch deutliche Gliederung. Die Beweglichkeit muss aber eine geringe sein, denn die verticale Klnft ist sehr seicht; ja, ich glanbe, dass, wenn ich znfällig ein Exemplar untersucht hätte, bei dem das collare nicht anfgehoben war, die Feststellung der Gliederung nicht ganz leicht gewesen wäre. Bei genauer Untersnchung zeigt sich jedoch, dass die Chitindecken der beiden Kopfteile nicht ineinander übergehen. Die Kluft setzt sich an den Seiten bis an die Antennengraben fort, und geht eigentlich noch viel weiter; oder mit anderen Worten, das collare ist an den Seiten sehr breit und bedeckt einen grossen vorderen Teil der Antennengrabe. Der Rand der Hinterseite der pars anterior ist hier nicht gut zu sehen, der der Vorderseite der pars posterior dagegen gut und breit chitinisiert. Weiter nach vorne bemerkt man einen inwendigen Höcker, das tuber frontale, das anssen in einer Vertiefung eine kleine dreieckige Spitze, einen mucro, trägt.

\section{Die weiter zu beschreibenden Köpfe sind ungegliedert.}

Der Kopf des Ctenocephalus canis (Curt.) (Taf. XII, Fig. 6) bietet uns ein Beispiel von einem sehr kräftigen tuber verticale, das wunderbarerweise aussieht wie das tuber postierticale der Macropsylla hercules Rothsch.; d. h. es scheint entstanden zu sein ans einer verticalen Falte, welche mit einer leichtgefärbten Chitinmasse ausgefüllt ist. Das Exemplar ist von Herrn Edw. Jacobson in seiner Wohnung in Semarang (Java) erbeutet worden. Ich besitze noch zwei andere Exemplare, und zwar aus Wien und ans Paris, aber keins von beiden zeigt das tuber so dentlich als eine Falte. Von diesem tuber ans verlanfen zwei inwendige, den Kopfseiten angedrückte Chitinleisten nach unten, und zwar vor und hinter der Antennengrube. Gerade über letzterer sieht man eine kaum bemerkbare Naht, welche als eine Verlängerung der scharfen Hinterkante des durchscheinenden Lappens erscheint, welcher die Vorderhälfte der Antennengrube bedeckt.

Weniger gross und fast kreisrund ist das tuber verticale (= der optische Durchschnitt durch den dicksten Teil der falx verticalis) bei Pulex irritans L. 
(Taf. XII, Fig. 7). Hier ist keine Andentung vorhanden, dass es aus einer ansgefüllten Falte entstanden ist. An den Kopfseiten befinden sich die allmählich dünner werdender Sichelhörner, welche sich vor und hinter den Antennengruben spalten. Über der falx verlänft dentlich eine Naht, welche den Anschein hat, als wäre sie eine etwas nach vorne verschobene Verlängerung der scharfen Hinterkaute des durchscheinenden Lappens, welcher die Vorderbälfe der Antennengrube bedeckt.

Beim Kopfe von Malacopsylla grossiventris Weyenb. (Taf. XII, Fig. 8) ist das tuber verticale kleiner, halbkreisförmig. Die Sichelhörner sind breit, dunkel, nud an der Antennengrube gespalten. Uber der falx verlänft eine sehr dentliche Naht, die anssieht als ob sie eine etwas nach hinten verschobene Verlängerung der scharfen Hinterkante des durchscheinenden Lappens wäre, weleher den vorderen Teil der Antennengrube bedeckt.

Als ich den Kopf von Parapsyllus longicornis Enderl. abbilden wollte, fand ich, dass dieser Typus von mir eben dem Herrn Dr. Enderlein zurïckgesandt war. So behalf ich mich mit einer Skizze des Kopfes einer anderen Parapsyllus-Art ans der Sammlung des Herrn N. C. Rothschild (Fig. A, S. 158), P. australiacus. Die falx verticalis ist deutlich, aber nicht schwer chitinisiert. Das tuber verticale (= der optische Durchschnitt durch den dicksten Teil der falx) ist noch kleiner als bei der vorhergehenden Art. Hier scheint keine die Antennengrube deckende Platte vorhanden zu sein. Weit nach vorn, fast an der vordersten Kopfspitze, befindet sich ein inwendiges tuber frontale, welches aussen, in einer Vertiefung, ein sonderbar geformtes Läppchen trägt, einen mucro.

Keine Spur von tuber verticale zeigt der Kopf von Dolichopsylla stylosus Baker (Taf. XIII, Fig. 9). Aber es ist ïberraschend, dass er einen hellen, wenig chitinisierten, Streifen besitzt, welchen ich als ein Rudiment einer Grube oder Falte oder Naht ansehe. Der Streifen, welcher von jeder Antennengrube nach dem Vertex verlänft, erreicht diesen offenbar nicht; sonst wäre dort wohl eine Unterbrechung der chitinösen Kopfdecke zu sehen. Die schuppige Chitinzeichnung ist vor diesem Streifen nach hinten und oben gerichtet, hinter demselben dagegen nach vorn und oben, wie in der Figur angegeben ist. Ein Teil der vorderen Hälfte der Antennengrube ist von einem durchsichtigen, schmalen Lappen bedeckt. Weit nach vorn, fast an der vordersten Spitze des Kopfes, bildet die Chitindecke einen Winkel, der nach unten (nach vorn) gerichtet ist. Bei anderen Floharten ist dieser Winkel höher am Kopfe angebracht, und weist dann nach oben (nach hinten). Es ist ein Mittelding zwischen einem mucro und einem protectum (siehe Tijdschrift, loc. cit.), denn es ist kein Dreieckchen, wie Fig. 3 zeigt, anch kein Schutzdächlein, das unter sich einen Raum besitzt, wie in der folgenden Fignr angegeben.

Keine Spar, weder einer falx, noch eines tuber verticale, noch einer Naht ist am Kopfe des Ceratophyllus hirundinis Curt. zn finden. (Da das mir vorliegende Exemplar von Ceratophyllus hirundinis Cnrt. beschädigt war, behelfe ich mich hier mit einer Abbildung einer änsserst naheverwandten Art, columbae, Taf. XIII, Fig. 10). Selbst die schuppige Chitinzeichnung verlänft schräg über den Antennengruben nach binten. Man bemerkt aber weit nach vorn ein wahres protectum, das wie ein Schutzdächlein einen kleinen Ranm birgt; es erinnert uns an ein collare; es ist aber nach unten (nach vorn) gerichtet. Es gibt indes Floharten, bei denen das protectum höher am Kopfe steht, in welchem Falle es nach oben (nach hinten) gekehrt ist (protectum incersum), so dass es einem wahren collare 
ähnelt. In beiden Fällen länft der Rand des Schutzdächleins ein wenig links und rechts über den Kopf hin, wie in der Fignr angegeben ist.

Demnach haben einen gegliederteu Kopf (caput fractum):

Chiropteropsylla aegyptius (Rothsch.), Ctenoplithalmus segnis (Schönh.), Hystrichopsylla talpae (Curt.), Ischnopsyllus elongatus (Curt.), Macropsylla hercules Rothsch., Nycteridopsylla pentactenus (Kolen.), Palaeopsylla gracilis (Tasch.), Stephanocircus dasyuri Skuse, Thaumapsylla breviceps Rothsch., Typhloceras poppei Wagn.

Von diesen besitzen ein tuber postierticale die zwei folgenden:

Macropsylla hercules Rothsch. und Stephanocircus dasyuri Skuse.

Von den Gattungstypen mit caput integrum nennen wir die folgenden, welche ein tuber verticale besitzen, das ans einer Falte, welche wieder ausgefuillt ist, entstanden zu sein scheint. Zugleich haben sie eine schwere falx:

Ctenocephalus canis (Curt.) und Moeopsylla sjoestedti Rothsch.

Eine schwere falx mit einem massiven tuber verticale haben die folgenden:

Archaeopsylla erinacei (Bouché), Hoplopsyllus anomalus Baker, Malacopsylla grossiventris Weyenb., Neopsylla bidentatiformis (Wagn.), Parapsyllus longicornis Enderl., Pulex irritans L., Rhopalopsyllus lutzi Baker, Spilopsyllus leporis (Curt.).

Die folgenden Arten besitzen ein schwaches tuber verticale und eine schwache falx oder eine Spur davon, und zwar in allen denkbaren Graden von Dentlichkeit :

Anomiopsyllus nudatus Baker(?), Echidnophaga ambulans Olliff, Goniopsyllus kerguelensis (Tasch.), Lycopsylla novus Rothsch., Odontopsyllus multispinosus Baker, Ornithopsylla laetitiae Rothsch., Pariodontis riggenbachi Rothsch., Pygiopsylla hilli (Rothsch.), Spalacopsylla bisoctodentatus (Kolen.).

Eine dentliche Naht verlänft über die falx bei :

Goniopsyllus kerguelensis (Tasch.) und Neopsylla bidentatiformis (Wagn.).

Eine zum Teil deutliche Naht ist vorhanden bei :

Anomiopsyllus nudatus Baker, Ctenocephalus canis (Curt.), Hoplopsyllus anomalus Baker, Malacopsylla grossicentris Weyenb., Pulex irritans L., Rhopalopsyllus lutzi Baker, Spalacopsylla bisoctodentatus (Kolen.).

Nur eine Naht finden wir bei :

Dolichopsylla stylosus Baker.

Keine Spuren von tuber verticale, falx, und Naht sind zn bemerken bei :

Ceratophyllus hirundinis Curt., Cerat. fasciatus (Bose), Cerat. sciurorum (Schrank), Cerat. penicilliger (Grube), Chaetopsylla globiceps (Tasch.), Coptopsylla lamellifer (Wagn.), Dasypsyllus perpinnatus Baker, Dermatophilus penetrans (L.), Hectopsylla psittaci Franenf., Listropsylla dolosus Rothsch., Loemopsylla chaeopis Rothsch., Hectopsylla pulex (Haller), Uropsylla tasmanicus Rothsch., Vermipsylla alacurt Schimk.

Wir treffen ein tuber frontale, mit oder ohne mucro oder listron (schanfelförmiges Läppchen) an bei :

Chaetopsylla globiceps (Tasch.), Listropsylla dolosus Rothsch., Neopsylla bidentatiformis (Wagn.), Parapsyllus longicornis Enderl., Rhopalopsyllus lutzi Baker.

Bei den folgenden Arten findet sich ein protectum oder ein protectum ähnliches Gebilde :

Ceratophyllus hirundinis Curt., Cerat. fasciatus (Bose), Cerat. sciurorum (Schrank), Cerat penicilliger (Grabe), Dasypsyllus perpinnatus Baker, Dolichopsylla stylosus Baker, Lycopsylla novus Rothsch., Odontopsyllus multispinosus Baker. 
Nur ein Genus zeigt ein protectum inversum, nämlich Moeopsylla sjoestedti Rothsch.

Ebenfalls ist nur ein Genus mit einem frontalen hufeisenförmigen Eindrucke versehen, und zwar :

Dermatophilus penetrans (L.).

\section{Ontogenetischer Teil.}

Wie die Nebelflecke, die Nebelsterne, die weissen, gelben, roten, einige veränderlichen und die verschwundenen oder dunkelen Sterne uns die Werdungsgeschichte der Himmelskörper erzählen, so belehren uns die oben abgebildeten und beschriebenen Flohköpfe über die Ontogenie dieses Körperabschnittes.

Betrachten wir die Figur 1, so können wir uns des Gedankens nicht erwehren, dass dieser Kopf bei der Larve dreiteilig, d.h. mit zwei Einkerbungen versehen war. Die hintere derselben muss während des Puppenstadiums stark chitinöse Ränder bekommen nnd sich darauf mit Chitinmasse ansgefüllt haben, während die vordere als Gelenk bestehen blieb.

Was die zweite Figur anbetrifft, so lehrt sie uns, dass die Larve ebenfalls einen dreiteiligen Kopf hatte, mit zwei tiefen Einkerbungen, wovon die vordere stark chitinöse Ränder bekam und als Gelenk blieb, während die hintere ebenso stark chitinöse Ränder erhielt, sich aber bald mit Chitinmasse so ansfüllte, das von einer Einkerbung keine Spur übrigblieb, sondern eine gewaltige in wendige Sichel gebildet wnrde. Ein feiner auswendiger Schlitz verrät aber deren Ursprung.

Fig. 3. zeigt uns den typischen Ischnopsyllus-Kopf. Hier können wir uns vorstellen, dass die Larve ebenfalls vor den Antennen eine Gliederung anfweist. Aber wir sehen am vordersten Kopfrorsprunge ein dreieckiges Gebilde, einen mucro. Es sieht ans, als ob es anfgeklebt ist, nnd in der Tat, bei einigen Exemplaren kann es abbrechen oder abfallen. Die Stelle, wo dieser angeheftet war, ist dann in den meisten Fällen angedentet, sei es dass sich dort eine sehr oberflächliche Einbuchtung befindet, oder eine kleine, gerade Strecke in der gebogenen Frontallinie zu sehen ist, oder aber ein Wölkchen von coaguliertem Eiweiss die Stelle verrät (siehe Tijdschrift voor Ent. vol. li. p. 94). Was ist die Bedentung dieses mucro? Das wissen wir nicht; vermutlich ist er ein Sinnesorgan. Woraus entsteht er; was war er bei der Larve? Auch das ist ein Rätsel, denn die Isclenopsylliden-Larven sind noch nicht bekannt. Vielleicht lehren uns die folgenden Köpfe etwas Näheres.

An Figur 4 sehen wir nur eine einzige Gliederung und schliessen darans, dass die Larve dieselbe Gliederung anfweisen muss.

Figur 5 zeigt uns, ausgenommen die Gliederung, welche sicher anch bei der Larve anwesend war, einen schönen kastanienförmigen mucro, welcher in einer seichten Vertiefung steht.(die ich bisweilen anch bei Ischnopsyllide fand-siehe oben). Da die Kopfdecke an dieser Stelle nicht dünner ist, als vor und hinter dem mucro, so springt sie inwendig etwas hervor, als wollte sie ein kleines tuber frontale bilden. Der Zusammenhang des mucro mit dem tuber frontale ist deutlich. Da die Larve uns unbekannt ist, so können wir nichts Näheres über den Ursprung dieses Gebildes mitteilen.

Der Kopf des Hundeflohs, Figur 6, ist sehr interessant, da er nns ein tuber verticale zeigt, welches dem tuber postierticale des Macropsylla hercules Rothsch. (Figur 1) so ansserordentlich gleicht, dass wir unwillkürlich den Schluss ziehen, die beiden tubera seien gleichen Ursprungs. Da nun das tuber verticale des 
Hundeflohs gerade an der Stelle angetroffen wird, wo die vorhergenannten Arten eine Kopfgliederung aufweisen, so schliessen wir, dass es früher, bei der Larve, eine Gliederung war, die aus gewissen Gründen verloren ging. Die Ränder, der Vorder- und Hinterfläche der Falte wurden stark chitinisiert, die Falte selbst füllte sich mit einer Chitinmasse aus, aber ein Teil der scharfen Kragenkante blieb als Naht über der Antennengrube sichtbar. Ist diese Betrachtung richtig, dann muss das tuber postverticale von Macropsylla hercules Rothsch. ebenfalls eine rudimentäre Gliederung sein.

Pulex irritans L. (Figur 7) besitzt einen Kopf, bei dem das tuber verticale keine Spur von Falte aufweist. Doch glaube ich, dass es, wie beim Hnndefloh, ursprünglich, also bei der Larve, eine Falte war, welche ihrerseits eine rndimentäre Gliederung sein muss. Bei genauer Untersuchung unterscheidet man noch eine Naht ïber der Antennengrube, als Rudiment einer Kragenkante.

Der Fig. 8 abgebildete Kopf hat ein starkes, obwohl relativ niedriges tuber verticale und eine breite $f a l x$, worüber eiue deutliche Naht von der einen Antennengrube zur anderen verläuft. Die Larve hatte hier gewiss eine Gliederung, welche aber nicht sehr beweglich war, also eine solche wie sie die ausgebildeten Flöhe Fig. 4 and 5 zeigen. Die Falte füllte sich ganz ans.

Sehr lehrreich ist der in Fig. A (S. 158) wiedergegebene Kopf. Erstens haben wir hier ein grosses tuber frontale, mit einer tiefen Einsenkung, worin ein länglichviereckiges Läppchen steht. Erinnert das Gebilde nicht an Figur. 5? Ist es nicht ein Übergang zwischen dem kleinen tuber frontale der Palaeopsylla gracilis (Tasch.), Fig. 5, und dem grossen Chaetopsylla globiceps Tasch.? Zweitens ist das tuber verticale der vorliegenden Art sehr klein; aber dadurch gleicht es ungemein dem tuber frontale von anderen Suctoria, speziell von Chaetopsylla globiceps, da diese nur eine seichte Vertiefung, keinen mucro oder list:on (wie Listropsylla), oder andersgeförmtes Läppchen trägt. Darum glaube ich, dass ein tuber frontale, mit oder ohne mucro, listron, oder andersgeförmtem Läppchen, ein Rudiment einer Falte und diese wieder eine rudimentäre Gliederung darstellt.

So muss ich wohl annehmen, dass bei der Larve zwei Kopffalten nachzuweisen sind, eine vor den Antennen, die andere nicht weit über den Mundteilen gelegen. Vielleicht ist die erste Falte selbst sehr tief, so dass sie beinahe eine Gliederung: darstellt ; denn woher soll sonst die breite braune falx herrühren?

Ebenfalls lehrreich ist Figur 9, welche den Kopf von Dolichopsylla stylosus Baker darstellt. Dieser besitzt über der Antennengrube einen hellen schmalen Streifen, welcher offenbar eine weniger chitinisierte Stelle ist, wie oben beschrieben. Dieser Streifen muss wohl eine seichte Falte bei der Larve sein. Eine zweite Eigentümlichkeit ist ganz vorn am Kopfe wahrzunehmen. Dort ist die Kopfdecke wie geknickt und wir dürfen vermuten, dass bei der Larve der Teil, welcher sich unter diesem Knicke befindet, in den darüber gelegenen Teil eingeschoben war oder werden konnte, mit anderen Worten, dass bei der Larve sich hier eine Gliederung befindet. Also gerade dort, wo bei den Larven eine Gliederung vorhanden ist, bildet sich ein tuber frontale, oder ein mucro etc.? Ja, und ich glanbe auch, dass dieser Knick und der mucro und das tuber frontale gleichen Ursprungs sind.

Figur 10 stellt den Kopf des Ceratophyllus columbae Steph. vor. Ich hätte ebensogut den Kopf einer anderen Ceratophyllus-Art abbilden können, denn die Köpfe sind einander aussergewöhnlich ähnlich. Die einzige Merkwürdigkeit an diesem Kopfe ist das Schutzdächlein (protectum). Sieht es nicht aus wie der Knick am vorigen Kopfe? Gewiss, aber es birgt einen kleinen Raum unter sich ; 
es hat eine scharfe Kante, welche sich noch ein wenig zn beiden Seiten des Kopfes erstreckt. Dieses protectum ist ganz gewiss eine rudimentäre Falte der Larve, oder eine rudimentäre Gliederung, was dasselbe ist. Es ähnelt anch, obschon umgekehrt, einem collare (Kragen, Hinterrand jedes Leibessegments der Flöhe).

Nun besitse ich eine Larve von Ceratoplyyllus fringillae (Walk.). Wer beschreibt meine Erregung als diese meine Vermutungen bestätigte! Wie dentlich die tiefe Falte, oder Gliederung, sich über den Mnndteilen hinzieht! Und gerade dort, wo bei der Imago das protectum hervorspringt ! Noch mebr! Gerade vor den Antennen erstreckt sich ebenfalls eine Falte (hier nur eine halbe Gliederung), also gerade dort, wo bei der Imago von Dolichopsylla der weisse Streifen verlänft, bei anderen Flöhen die Naht, die falx oder die Gliederung sich befindet ! :

Absolut sicher steht die Tatsache, dass die supra-antennale Gliederung, falx, Naht, Streifen, etc., gemeinsamen Ursprnngs, homolog sind.

Ist dasselbe mit dem tuber postverticale der beiden erstgenannten Flohgattungen der Fall? Diese Frage ist entschieden zu bejahen, denn bei beiden Arten befindet sich das tuber in der Mitte der pars posterior, zwischen den zwei "augenähnlichen Organen."

Und wie steht es mit dem tuber frontale, dem mucro, etc.? Ich glanbe, dass wir mit Vertrauen annehmen können, dass alle diese Gebilde gemeinsamen Ursprungs, also homolog sind. In den meisten Fällen befinden sie sich $\mathrm{z}$ wischen dem ersten und zweiten " augenförmigen Organe" (von der Stirnkante ab gerechnet). Es giebt zwei Ausnahmen : der mucro der Ischnopsyllidae ist gerade vor oder selbst unter dem ersten "angenförmigen Organe" gelegen ; aber wenn man bedenkt, dass die Stellnng dieser Organe kleinen Schwankungen ausgesetzt ist, dann nimmt man es nicht so genau damit. Noeopsylla sjoestedti Rothsch. besitzt ein sehr hoch, gerade vor dem Vertex, gelegenes protectum inversum. Ich erinnere mich nicht, wie dieses sich gegenüber den "angenförmigen Organen" verhält; denn ich machte darüber keine Notizen ; ich glanbe aber, dass es obue Bedenken als homolog mit den anderen genannten Frontalorganen angesehen werden kann.

\section{Phylogenetisch-Biologischer Teil.}

Bevor ich nun zu dem ïbergehe, was mich das Studium der Flohköpfe gelehrt hat, muss ich noch einige Grundsätze erwähnen.

Sowohl das Studium der Acari, als das der Suctoria hat mir in erster Linie gezeigt, dass die Weibchen die primitive Organisation fast immer weit besser bewahrt haben als die mehr spezialisirten Männchen. Darum sind z. B. die $q \uparrow$ der Gattungen Ceratophyllus und Ischnopsyllus so schwer voneinander zu unterscheiden. Aus diesem Grunde basiren sich meine Betrachtungen und Klassifikation nur auf die Weibchen.

Was die Artkennzeichen betrifft, so ist es bekannt, dass bei schwer zu unterscheidenen Arten nor die hintersten Teile (Gonapophysen, etc.) benutzt werden können. Es ist darum logisch, dass man für die Unterscheidung von höheren Gruppen: Genera, Familia, Superfamilia, wenn möglich Unterschiede in mehr nach vorn gelegenen Teilen ins Ange fasst.

Wenn wir nun zu dem eigentlichen Gegenstande unseres Studiums übergehen, so müssen wir zunächst fragen, welche Eigenschaften die Flöhe allmählich bekommen oder verloren haben in Verbindung mit ihrer Lebensweise oder besser als Folge derselben. 
A. Die Länge.-Wahrscheinlich waren die Vorfahren der jetztlebenden Flöhe Parasiten der ersten, kleinen, nervösen und daher sehr beweglichen Sängetiere, nämlich der Allotheria, Monotremata, Marsupialia, Insectivora und Chiroptera mit sehr dichter Behaarung. Die Tierchen müssen wohl eine lange, schmale Form gehabt haben und änsserst gewandt gewesen sein, um durch das Dickicht von Haaren eilen und dem Gekratze der Wirte entfliehen zu können. Ich sehe darum in langgestreckten Flöhen primitive Formen.

Wahrscheinlich durch fortgesetzten Parasitismus auf grösseren, ruhigeren Säugetieren, wurden die Flöhe selbst, da sie weniger beunruhigt wurden, auch allmählich weniger beweglich, weniger schlank.

Auch ist es denkbar, dass einige Floharten der schon kürzeren Formen sich gewöhnten, sich an gewissen blutreichen Stellen der Wirte festzusaugen und dann dort zu verharren. Wird solch ein einziger Stich ausgeübt, während der Wirt schläft oder flüchtet oder frisst, dann ist der Parasit fast ganz sicher nicht gestört zu werden, kann sitzen bleiben und wird im Laufe des Zeiten stets kürzer und fettreicher.

Lange Flöhe sind z. B.: Ischnopsyllus, Ctenophthalmus, Hystrichopsylla ; kurze: Pulex, Archaeopsylla, Ctenocephalus; sehr kurze: Dermatophilus, Echidnophaga, Hectopsylla.

B. Die Beweglichkeit und die Sprunqfähigkeit.-Wir können uns vorstellen, dass die ersten Flöhe gewöhnliche Gangbeine hatten und gar keine Sprungfähigkeit besassen. Es ist leicht zn begreifen, dass sie diese Fähigkeit allmählich erwarben, um dem Gekratz zu entgehen, oder besser, um nach dem Absterben des Wirtes so bald wie möglich einen anderen Wirt erreichen zu können. Ursprüugliche Flöhe müssen daher sprunganfähige Beine haben, oder nur sehr kleine Sprünge machen können. Chiropteropsylla, Ischnopsyllus, Nycteridopsylla und Thaumapsylla können hier genannt werden. Wir können anch annehmen, dass die Flöhe der Fledermäuse sekundär schlechte Springer geworden sind, weil ihre wirte Lufttiere und ziemlich hilflos sind. Viel bessere, ja gewaltige Sprüge machen gerade die mittellangen Flöhe wie Archaeopsylla, Ctenocephalus und Pulex. Und selbstverständlich machen die Fellhocker, wie man die sonderbar spezialisierten Dermatophilus, Echidnophaga und Hectopsylla nennen kann, gar keine Sprünge mehr, vielleicht abgesehen von den Weibchen, ehe sie sich festgesetzt haben, und den Männchen.

C. Die Behaarung und Beborstung.-Mir kommt es vor, als ob die ursprünglichen Flöhe dicht behaart oder besser beborstet waren, oder wurden. Denn waren die Vorfahren schon beborstet, dann war eine neue Beborstung nicht nötig. Waren sie aber stark behaart, so mnsste sich wohl die Behaarung allmählich in eine Beborstung umwandeln, d.h. jedes Haar wurde steifer. Steife Haare oder Borsten siud gewiss solchen zwischen Haaren lebenden und fortwährend beunruhigten Tieren vorteilhaft; sie setzen den Besitzer instand besser fortzugleiten. Hystrichopsylla, Macropsylla, Dotichopsylla, Goniopsyllus, Listropsylla, Neopsylla, Odontopsyllus (?), Parapsyllus, sind alle dicht oder ziemlich dicht beborstet.

Die Beborstuug geht allmählich verloren, wenn die Tiere weniger beweglich werden, d. b. weniger zwischen den Haaren der Wirte forteilen. Die Mehrzahl der Flöhe ist denn anch wenig beborstet, $d$. h. jedes Tergit oder Sternit trägt etwa zwei oder nur eine Querreihe von Borsten.

Und endlich sind die Fellhocker: Dermatophilus, Echidnophaga und Hectopsylla, fast unbeborstet, kahl.

D. Die Bedornung der Beine.-Je beweglicher die Flöhe auf Ihren Wirten 
sind, je schwerer sind die Beine bedornt und desto mehr ist die Sohlfläche des 5. Tarsalgliedes feinbehaart. Mit Dornen bezeichne ich dornförmige Haare, also bewegliche Gebilde.

a. Die Procoxae.-Thaumapsylla, Ctenophthalmus, Hystrichopsylla sind Beispiele von Flöhen mit polystichen (vielreihig behaarten) Procoxae. Merkwürdigerweise auch Dermatophilus. Mesostich sind die Procoxae z. B. von Archaeopsylla, Parapsyllus und Spilopsyllus. Wenig beborstet (oligostich) ist z. B. die Procoxa bei Chaetopsylla, Malacopsylla und Vermipsylla. Fast kabl ist die Procoxa von Echidnsphaga und Lycopsylla.

b. Die Tibiae.-Eine geschlossene Reihe von Borsten findet man an den Pro-, Meso- und Metatibiae der Chiropteropsylla und Ctenophthalmus, an den Pro- und Mesotibiae von Thaumapsylla, an der distalen Hälfte der Protibia der Hystrichopsylla und an der distalen Hälfte der Metatibiae der Thaumapsylla. Dies sind alle ziemlich primitive Flöhe. Einen Ubergang zu den "gewöhnlichen Flöhen " bildet Stephanocircus. Dieser Floh hat eine Protibia mit 6 Einschnitten, welche 2, resp. $2,3,3,3$ und $;$ Borsten tragen, eine Meso- und eine Metatibia mit 7 Einschnitten, welche 2, resp. $2,3,3,3,3$ und $\tau$ Borsten tragen, sodass es scheint, als ob die Tibien mit einer geschlossenen Reihe von Borsten versehen wären. Auch Macropsylla ist hierin primitiv. Die Protibia hat 9 Einschnitte und Reihen von 3 und 4 Borsten, die Mesotibia 13 Einschnitte und Borstenpaare, und die Metatibia 14 Einschnitte, in denen abwechselnd zwei und drei Borsten stehen. In der Regel jedoch haben die Tibien nur 6 bis 10 Einschnitte, welche mit Borstenpaaren versehen sind. Wenig Einschnitte und wenig Borsten kennzeichnen spezialisierte Flöhe. So besitzen die folgenden Flöhe die daneben angegebene Zahl Einschuitte an drei Tibien :-

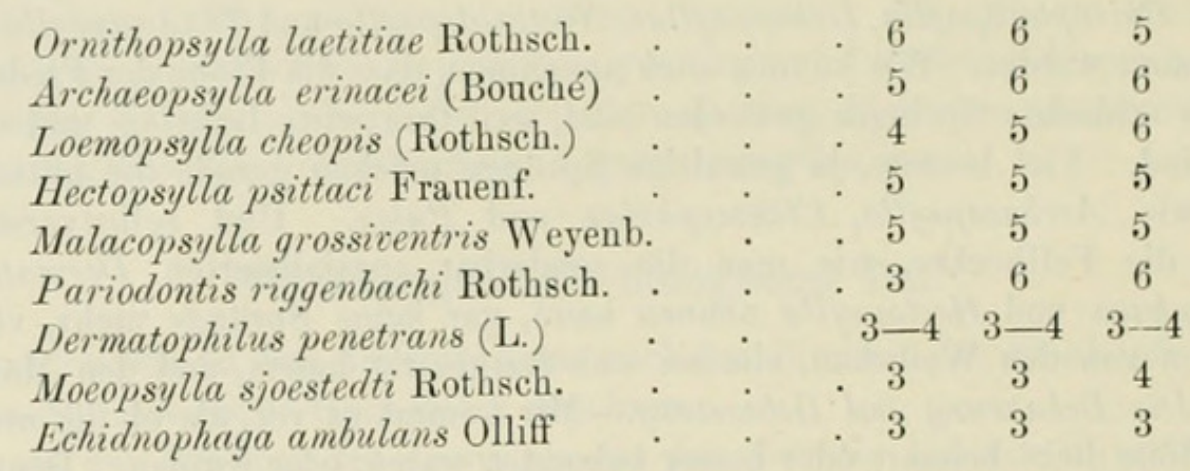

c. Das fünfte Tarsenglied.-Dieses ist ebenfalls ein lehrreiches Objekt. Es kann an seiner Plantarfläche laterale, subbasale, subapicale und plantare Borsten oder Haare tragen. Je mehr von jeder Sorte, je primitiver die Art. Wenn die plantaren in grosser Zahl vorhanden sind, so sind es immer sehr feine Härchen, womit die ganze Sohle oder nur die distale Hälfte derselben besetzt ist. Sie dienen gewiss dazn, das Ausgleiten zn verhiudern. Ganz unbehaart ist die Sohle (abgesehen von den 1 oder 2 subapicalen Borsten) bei den folgenden Arten, welche entweder wenig oder gar nicht beweglich sind :

Dermatophilus penetrans (L.), Echidnophaga ambulans Olliff, Hectopsylla psittaci Franenf., Lycopsylla novus Rothsch., Malacopsylla grossiventris Weyenb., Ornithopsylla laetitiae Rothsch.

E. Der Besitz von Ctenidia.-Mit Ctenidia bezeichne ich nur die unbeweglichen, meist dnnkel gefürbten Zinken, welche die Ränder von Kopf, Thoracaloder Abdominalsegmenten zieren. Also sind die platten, schwarzen Borsten 


\section{$(145)$}

am Vorderkopfe des Ctenophthalmus segnis (Schönh.) und am 7. Tergite der Nycteridopsylla pentactenus (Kolen.) Pseudoctenidien.

Die Ur-Suctoria können keine Ctenidien gehabt haben. Diese müssen allmählich an den nach hinten gerichteten Kopf-, Thorax- und Abdominalrändern entstanden sein, und zwar sehr wahrscheinlich aus denselben Gründen, warum die ursprüngliche Behaarung sich in eine Beborstung umwandelte, nämlich um dem Insekt zu gestatten, schneller zwischen den Haaren der Wirte fortzueilen.

Wenn diese Meinung richtig ist, dann müssen wir bei den ersten Flöhen eine Steigerung der Ctenidienzahl, oder der Zinkenzahl in homologen Ctenidien aufweisen können. Wir können auch begreifen, dass mit der Abnahme der Beweglichkeit der Parasiten, die Zahl der Ctenidien, oder der Zinken in homologen Ctenidien, sich wieder verminderte. Ist diese Meinung richtig, dann ist schwer zu entscheiden, ob eine geringe Zahl von Ctenidien, oder von Zinken in homologen Ctenidien, ein primitives oder ein secundäres Merkmal ist. In solchen Fällen müssen andere Kennzeichen mithelfen, z. B. die Länge, die Beborstung, etc.

Die folgenden Flöhe tragen keine Ctenidien :-

* Chaetopsylla globiceps (Tasch.), Coptopsylla lamellifer Wagn., ! Dermatophilus penetrans (L.), ! Echidnophaga ambulans Olliff, Goniopsyllus-kerguelensis (Tascb.), ! Hectopsylla psittaci Franenf., Loemopsylla cheopis Rothsch., Lycopsylla novus Rothsch., *Malacopsylla grossiventris Weyenb., Moeopsylla sjoestedti Rothsch., Ornithopsylla laetitiae Rothsch., Parapsyllus longicornis Enderl., Pariodontis riggenbachi Rothsch., Pulex irritans L., Rhopalopsyllus lutzi Baker, *Vermipsylla alacurt Schimk.

Zum Teil sind es spezialisirte, zum Teil sehr spezialisirte Flöhe. Keine Art kann primitiv genannt werden. Die mit einem* bezeichneten sind im Begriff Eiersäcke zu werden; die mit einem ! gekennzeichneten sind es schon.

F. Die comprimierte Leibesform.-Diese Leibesform ist entschieden die best geeignete zum schnellen Forteilen zwischen den Haaren des Wirtes. Ur-Suctoria waren wahrscheinlich cylindrisch oder etwas abgeflacht (wie die meisten freilebenden Insekten). Die Mehrzahl der jetztlebenden Suctoria besitzt eine comprimierte Leibesform. Mehr spezialisierte Flöhe, wie z. B. Pulex irritans L., sind, wenn pregnant, schon "etwas dick" zu nennen. Noch mehr ist dies der Fall bei Chaetopsylla globiceps (Tasch.), und besonders bei dem wenig beweglichen Weibchen von Vermipsylla alacurt Schimk. und Malacopsylla grossiventris Weyenb. Missgestaltet können die Weibchen der Fellhocker Dermatophilus penetrans (L), Echidnophaga ambulans Olliff und Hectopsylla psittaci Franenf. genannt werden. Die Monstrosität erreicht den Gipfel in der Kugelgestalt von Dermatophilus caecata Enderl.

G. Die Chitinisierung.-Solche zwischen Haaren lebende und forteilende, fortwährend beunruhigte, und dem Kratzen von Seiten des Wirts ausgesetzte Quälgeister müssen natürlich stark chitinisiert sein. Weiche Hautpartien sind bei Flöhen gefährlich. Aber sobald Flöhe sich gewöhnten, an gewissen blutreichen Stellen stille zu verharren, oder auf Tieren zu leben, die nicht kratzen können, wie die Ungulata, war die Möglichkeit vorhanden, dass die Chitinplatten des Abdomens weicher, und die Hant zwischen diesen Platten dehnbarer wurde. Solche Flöhe sind z. B. Chaetopsylla globiceps (Tasch.), Vermipsylla alacurt (Schimk.) und Malacopsylla grossiventris Weyenb. Die Chitinisierung ist verhältnismässig am meisten reduziert, die Dehnbarkeit daher am grössten, bei den Fellhockern und erreicht ihren Höhepunkt in Dermatophilus caecata Enderlein, bei welchem das 
ganz weiche und dehnbare Abdomen sich ringsum über Thorax, Beine und Kopf nach vorn umbiegt, sodass diese Teile ganz eingeschlossen werden.

H. Die Vergrösserung der frontalen Region.-Wir können uns leicht vorstellen, dass die vor den Antennen gelegene Partie, das Vorderhaupt oder die frons, sich als bohrendes, das Dickicht von Haaren durchdringendes Organ stark entwickelte. Ich sehe denn anch in solch einer stark nach vorn gerichteten frons einen primitiven Zustand. Siehe z. B. Figuren, 2, 3 und 4. Das will jedoch nicht sagen, dass ich die Ischnopsyllidae für die primitivsten der jetztlebenden Flöhe halte. Denn andere Flöhe können in vielen anderen Punkten noch viel primitiver sein. Je mehr sich die Schnelligkeit der Fortbewegung zwischen den Haaren der Wirte verminderte, desto mehr nahm die frons wieder in der Entwickelung ab. Hiervon sind die Figuren 1, 5 und 6 gute Beispiele. Dieser Zuriickgang ist am meisten bei den Fellhockern zu bemerken. Von diesen kann man wohl sagen, dass sie keine so typisch vorausstrebende frons haben, wie die anderen Flöhe.

I. Die Richtung der Antennen.-Antennen können als Gefühls-Gliedmassen nur nach vorn gerichtet gedacht werden. So bald aber die Vorfahren der Flöhe nicht mehr frei lebten, sondern für immer auf Säugetieren schmarotzten, konnten sie ihre Antennen nicht mehr nach vorn gerichtet halten, ohne sie zu verletzen. Die Folge war, dass sie sich angewöhnten, diese Gliedmassen stets nach hinten und unten zu halten, wobei zugleich die Bedeutung der Antennen als Gefühlsorgane verloren ging. Alle jetzt lebenden Flöhe halten ihre Antennen nach hinten und unten.

J. Die Stelle der Antennen.-So bald die Antennen ihre Bedentung als Gefühlsorgane einbüssten, war ihre Stellung am vordersten Teile des Kopfes auch keine unbedingt notweudige mehr. Sowohl aus diesem Grunde als auch durch die enorme Entwickelung der frons, wurden sie sozusagen nach hinten verschoben, wert hinter die Mundteile, nicht immer hinter die Ocellen (siehe u. a. Figuren 2, 3, 4 und 10). Diese einmal eingenommene Stelle hat sich bei den jetztlebenden Flöhen erhalten.

K. Die Bildung der Antennengrube.-Wenn Blutgefässe fest gegen Knochen lagern, bilden sich in diesen bisweilen tiefe Gruben. So kann man sich auch vorstellen, dass die Haltung der Antennen nach hinten und unten und zugleich fest gegen die Seiten des Kopfes und der Propleuren die einzige Ursache der Entstehung von Antennengruben war. Diese scheinen sich also zur Zeit gebildet zu haben, als die Suctoria noch keine besonders harte Chitindecke hatten.

L. Die Verkürzung der Antennen.-Sobald die Antennen ihre Bedeutung als Gefühlsorgane veloren hatten, war ihre ursprüngliche Länge nicht mehr unbedingt nötig und begannen sie sich zu verkürzen. Ob die Antennen schon ziemlich kurz waren, als die Vorfahren der Suctoria zum Parasitismus anf Sängetieren übergingen, ist unmöglich nachzuweisen. Jedenfalls sind die längsten Antennen der jetztlebenden Flöhe kurz zu nennen. In der Ruhe reichen sie nicht weiter als ungefähr bis zur Mitte der Propleuren, auf die sie sich, wie man es ausdrückt, "fortsetzen." In Wahrheit betrachte ich gerade diese Fortsetzung als ein primitives Verhalten, und das Kürzerwerden der Antennen, zugleich mit dem Verschwinden dieser Fortsetzung der Grube als ein secundäres Merkmal. Merkwürdigerweise haben die Mehrzahl der Männchen in den langen Antennen primitivere Kennzeichen als die Weibchen (Fig. 12). Es sind wohl keine Gefühlsorgane, sondern ausgezeichnete Riechorgane, auf denen hunderte von zierlichen, spatelförmigen Riechhärchen stehen. Die Männchen sind im Stande ihre Antennen aufzuheben, sodass diese als Öhrchen 
anfrecht stehen. In dieser Haltung sind die Antennen in fortwährender zitternder Bewegung.

Im Vergleich zu den Männchen besitzen die Weibchen die kürzeren Antennen. Da die Antennen wohl bei allen Suctoria dieselbe physiologische und biologische Bedeutung und ansserdem alle Suctoria wohl so ziemlich dieselbe Lebensweise haben, so sollte man meinen, dass die Antennen bei allen Suctoria dieselbe nnveränderliche Gestalt anfwiesen, wie sie bei keiner anderen Tiergruppe wiederzufinden ist. Im grossen und ganzen ist dieses auch der Fall. Die Flohantenne ist sehr characteristisch. Sie besteht: (1) ans einem umgekehrt kegelförmigen, an der Basis etwas ausgehöhlten scapus; (2) aus einem in dieser Aushöhlung liegenden napf- oder becherförmigen pedicellus, und (3) aus einem im Boden desselben angehefteten zehngliederigen kenlenförmigen funiculus. Das erste Glied des funiculus ist in seiner proximalen Hälfte immer sehr dünn. Ich werde es in der Folge das Stielchen oder den petiolus nennen, während ich die nenn übrigen Glieder des funiculus zusammen als Keule, clava bezeichnen will. In der schon mehrmals genannten Tijdschrift beschrieb ich die Antennen als elfgliederig. Bei einem sehr günstig conservirten Objekte, dass ich Herrn Alfons Dampf in Königsberg i. Pr. verdanke, konnte ich feststellen, dass der petiolus ein Glied ist, das bis jetzt übersehen wurde (Fig. 12).

Warum ist der petiolus als Glied übersehen worden? Erstens weil bei Antennen in der Ruhe die Lage des Stielchens für eine exacte Beobachtung ungünstig ist, nnd zweitens weil bei den meisten Antennen der petiolus im zweiten Gliede des funiculus eingesenkt ist, also selbst in günstiger Lage schwer zu sehen ist.

Wenn nun von dieser Einförmigkeit der Antennen eine "Ausnahme" stattfindet, so müssen wir wohl annehmen, dass diese Abweichung ein gutes Merkmal für natürliche Gruppen ist. In der Tat, die clava weisst zwei typische Formen bei den Weibchen der Flöhe auf.

Die erste Form ist eine etwas länglich ovale oder elliptische (Fig. 13, 14, 19). Ich betrachte sie als die primitivere, denn erstens ist sie länger als die zweite Form, zweitens haben die Männchen dieser Weibchen noch längere clavae, und drittens sind die neun Glieder der clava ziemlich gleichgestaltet, nicht besonders spezialisiert.

Die zweite Form ist eine mehr runde (Fig. 15-18): sie ist zweifellos die mehr spezialisierte, weil sie noch kürzer ist als die erste; die Männchen dieser Gruppe keine längeren clavae haben als die Weibchen; und weil der freie Teil des ersten Gliedes, oft auch eins oder der zwei folgenden Glieder mandolinenförmig gestaltet ist. Phylogenetisch gesprochen ist diese clava jünger als die vorige.

M. Die Verwachsung einiger Antennenglieder.-Mit der Verkürzung geht Hand in Hand das Breiterwerden der Glieder und ihrer Ansatzflächen. In der Tijdschrift voor Entomologie, vol. li., p. 97 (1908) teilte ich mit, dass ich die "Stielchen" der verschiedenen Glieder gefunden hätte.

Ich muss meine Mitteilungen darüber leider verbessern. Nur der Petiolus ist in seiner proximalen Hälfte dünn ; alle anderen Glieder sind mit breiter Basis am vorhergehenden Gliede befestigt. Nur bei den Männchen gewisser Gattungen, z. B. Ceratophyllus, könnte man sagen, dass auch das letzte Fühlerglied mit dünner Basis angeheftet sei. Nimmt man nun an, dass ein Autennenglied nur als solches bezeichnet werden kann, wenn es mit enger Basis am vorhergehenden Gliede befestigt ist, dann bestehen die Antennen der Suctoria aus nur zwei resp. höchstens 
drei Gliedern. Das erste Glied ist dann scapus und pedicellus zusammengenommen, das zweite derfuniculus. Nur bei den Männchen der Ceratophyllus-Grappe kann man das letzte Antennenglied als drittes bezeichnen.

Wenn daher die Mehrzahl der Entomologen noch annehmen, dass die Antenne der Suctoria nur ans drei Gliedern besteht, nämlich ans scapus, pedicellus und funiculus, so handeln sie inconsequent. Denn erstens sind anch scapus und pedicellus breit miteinander verbunden, und zweitens werden doch bei anderen Insekten, die breit miteinander verbundenen Glieder als solche anerkannt, z. B. bei der grossen Kïferabteilnug der Clavicornia und anderen.

Nun kann man mir einwerfen, dass in der genannten Tịjdschrift gesagt ist, dass ich die "Stielchen" der neun Glieder der clava gesehen habe und sie abbilde. Ich antworte hierauf, dass die Gebilde, welche ich für "Stielchen " angesehen, in Wirklichkeit vorhanden sind, aber dass ich mich damals in der Deutung derselben geirrt habe. Vielleicht sind es Sinnesorgane, welche sich an den Vorder- und Hinterseiten der scheibenförmigen Antennenglieder befinden, wo sie am besten gegen jede Verletzung geschützt sind.

Ich sagte oben, die Antennen beständen aus höchstens zwölf Gliedern. Andererseits giebt es Antennen, welche weniger als zwölf Glieder enthalten. Schon die Antennen von Macropsylla und Stephanocircus zeigen eine Tendenz, das erste Glied der ovalen clava zu verlieren. Es ist kleiner als die übrigen Glieder (Fig. 1, 2).

Die Antenne von Hectopsylla (Fig. 19) besteht aus vier Gliedern, nämlich dem scapus, dem pedicellus, dem petiolus, und den zusammengewachsenen Gliedern der ovalen clara. Diese selbst zeigt nur sieben Einschnitte zwischen acht Läppchen, was auf acht ursprüngliche Glieder hinweisst; also ist ein Glied spurlos verschwunden. Frage: Welches Glied? Antwort: Das ist vorläufig nicht festzustellen; "Ubergangsformen " kennen wir noch nicht.

Die Antenne von Ctenocephalus (Fig. 16) hat neun Glieder, nämlich den scapus, den pedicellus, den petiolus, ein ans vier ursprünglichen Gliedern verwachsenes Glied, und die fünf distalen freien Glieder der runden clava.

Bei Pulex treffen wir (Fig. 17) eine Antenne von zehn Gliedern, bestehend ans dem scapus, dem pedicellus, dem petiolus, fünf freien Gliedern und einem aus den drei distalen Gliedern der runden clava verwachsenen Gliedern. Wir müssen noch hinzufügen, dass das dritte Glied der clava im Begriff ist sich rückzubilden ; es ist bisweilen schwer zu sehen.

Fast unverkennbar ist die Verwachsung bei der Antenne der Echidnophaga gallinaceus (Westw.) (Fig. 18). Hier sind zu unterscheiden : scapus, pedicellus, petiolus, ein vermutlich aus zwei ursprünglichen Gliedern verwachsenes Glied, und ein ans vermutlich sieben ursprünglichen Gliedern verwachsenes Glied, also zusammen fünf Segmente. Züblt man die Furchen an der Hinterseite der Antenne (in der Figur getüpfelt), dann kommt man auf sieben Glieder. Ich bin mir nicht ganz im Klaren hierüber.

Wie man aus den Figuren 12 bis 19 und aus dem oben Gesagten ersehen kann, ist die Verminderung der Gliederzahl, sowohl durch Verwachsung als durch Verschwinden, bei beiden Formen von Antennen möglich, und ich glaube, dass wir es hier mit einer Convergenz-Erscheinung, nicht mit Verwandtschaft zu tun haben, dass also Hectopsylla nicht mit Echidnophaga verwandt ist.

N. Das Fehlen von zusammengesetzten Augen._-Es kann uns nicht verwundern, dass die Facetten-Augen, angenommen sie sind je vorhanden gewesen, spurlos verschwunden sind. Was sollten Insekten, die das freie Leben aufgegeben haben, 
noch mit zusammengesetzten Angen machen, die ja befähigen, nach verschiedenen Richtungen hin gut zu sehen?

O. Die Reduction der einfachen Augen.-Nur die einfachen Angen, Dunkelangen oder Ocellen, sind übriggeblieben und zwar anch nur bei denjenigen Suctoria, welche noch oft genug das Tageslicht eben sehen können, z. B. beim Hundefloh, Menschenfloh, ja selbst bei allen Fellhockern. Aber bei den Parasiten von in Höhlen oder hohlen Bäumen lebenden Säugetieren sind selbst die Dunkelangen rudimentär geworden oder fehlen ganz, z. B. bei den Flöhen von Maulwürfen und Fledermänsen. Hierans ersehen wir, dass der Besitz oder die Abwesenheit von Dunkelaugen oder Ocellen abhängig ist von der Lebensweise der Wirte, dass sie also keine Kennzeichen von höheren Gruppen, sondern höchstens von Genera oder Species sein können.

P. Die Richtung der Mundteile.-Als Folge der Lebensweise, ein fortwährendes Schiessen durch ein Dickicht von Haaren, richteten sich die Mundteile nach hinten, wurden zwischen die Vorderbeine aufgenommen. Dadurch wurden sie vollkommen gegen Verletzung geschützt. Durch die Entwickelung der frons nach vorn rückten anch die Mundteile scheinbar, oder anch in Wirklichkeit, nach hinten (vergl. z. B. Fig. 3). Aber je mehr die Schnelligkeit der Bewegung in dem Haargebüsch abnahm, desto mehr verminderte sich die Entwickelung der frons, desto mehr schoben sich die Mundteile wieder nach vorn, ja veränderten auch ihre Richtung. Sie nahmen wieder allmäblich eine ursprünglichere Lage an. So findet man z. B. bei Pulex die Mundteile fast senkrecht auf der Kopfachse stehen. Und bei den Fellhockern, die jede Bewegung anfgegeben haben, sind die Mundteile fast ganz vorn am Kopfe gelegen und auch fast gerade nach vorn gerichtet. Jordan und Rothschild haben schon (Revis. of Sarcops. p. 23, 1906) hierauf hingewiesen.

Q. Die Beschaffenheit der Mundteile.-Diese sind, wie bekannt, sogenannte stechende und sangende, da die Flöhe sich anpassten, von Säugetier-, später auch von Vogelblut zu leben. Es ist die Frage berechtigt, ob die Mundteile schon stechend und saugend waren, als die Ur-Suctoria zum Parasitismus anf Saugetieren übergingen. Die Frage ist vielleicht erst sicher zu beantworten, wenn wir etwas mehr von der Verwandtschaft der Suctoria wissen. Aber bis jetzt scheinen die Ansichten darüber auseinander zu gehen und scheinen mir immer dunkler zu werden. Alle jetztlebenden Suctoria haben im grossen und ganzen die verschiedenen Mundteile gleichgestaltet. Vom Labrum ist noch zu wenig bekannt, $\mathrm{nm}$ sich darüber zu rerbreiten. Die Epipharynx oder der sogenannte Stechapparat ist bei allen Suctoria vollkommen gleichgestaltet, besitzt nur bei der einen Gattung zahlreichere nach vorn gerichtete Sinnesorgane in der Gestalt von winzigen Höckerchen in der distalen Hälfte als bei der anderen. Da, wie unten erörtert werden soll, die Mandibeln die eigentlichen die Hant durchbohrenden Organe sind, so ist die Epipharynx distal auch nicht spitz, wie die Stechorgane von Mücken, Stechfliegen, Wespen und Bienen, sondern stumpf abgerundet. Die Epipharynx besitzt hinten eine offene Rinne, wirkt daher capillarisch, wie die Mandibel der MyrmeleontidenLarven. Die Capillarität in solchen dünnen Rinnen oder Röhren beträgt mehrere Centimeter. Es ist daher nicht nötig anzunehmen, dass die Epipharynx und die Mandibeln zusammen eine Saugröhre darstellen. Die Einförmigkeit der Epipharynx in der ganzen Ordnung zeigt, dass sie keinen systematischen Wert hat.

Die Mandibeln dienen erstens als Scheide für die Epipharynx, denn diese muss in erster Linie nnverletzbar sein. Zweitens sind sie die eigentlichen die Hant durchbohrenden Organe, denn sie sind sägeförmig, sind also besser Säge- als 
Stechapparat za nennen. Dienen sie nur zum Durchsägen der Hant, so können die Zähne klein nnd fein und die Mandibel selbst ziemlich schwach and durchsichtig sein. Dienen sie dagegen anch zur Befestigung, dann müssen die Zähne grob und stark sein, wie bei den Ixodidae. In diesem Falle sind die Mandibeln selbst lang, stark und braun chitinisiert (Spilopsyllus und die Fellhocker). Diese Beschaffenheit der Mandibel ist also eine Folge der Lebensweise, kann daher anf Verwandtschaft weisen, oder aber mag anch eine Convergenz-Erscheinung sein.

Die Maxillen sind bei allen Suctoria ziemlich gleich gestaltet. Sie bestehen aus fünf Gliedern. Das erste Glied, das ans einer Verwachsung von cardo, stipes und malae entstanden gedacht werden kann, hat von der Seite gesehen eine ziemlich dreieckige Form. Wir nennen die Seiten : die obere, die vordere und die hintere, und finden zwei Entwickelungsextreme. Entweder ist die obere Seite die kürzeste, sodass die vordere und hintere zusammen eine scharfe, nach unten gerichtete Spitze bilden; oder die hintere ist die kürzeste, sodass diese eine nach hinten gerichtete Basis darstellt. Im ersteren Falle ist die Basis, im zweiten Falle die Spitze unter dem Rande der Gena versteckt. Wovon ist diese Gestaltung ahhängig? Darauf bleibe ich die Antwort schuldig; aber es ist merkwürdig, dass die ziemlich primitiven Ischnopsyllidae und die sehr spezialisirten Fellhocker beide Maxillen besitzen, welche mit kurzer Basis nach hinten gerichtet sind, sodass in den Beschreibungen von stumpfen Maxillen die Rede ist; wäbrend die mittleren Suctoria spitze Maxillen haben. Es scheint also, dass die stumpfen Maxillen die primitiveren sind. Die Maxillarpalpen sind in der ganzen Ordnung gleichgestaltet, mögen sie kürzer oder länger sein.

Das Labinm besteht aus einem einfachen Mentum und zwei Palpen. Diese Labialpalpen dienen hauptsächlich als Scheide für die Mandibeln. Darum sind die Labialpalpen vorn und hinten ziemlich stark chitinisiert und symmetrisch, wenn die Mandibeln ziemlich weich sind (primitives Merkmal). Sie sind dagegen nur vorn chitinisiert und hinten häntig, also asymmetrisch, wenn die Mandibeln stärker sind (secundäres Merkmal). Und endlich ganz häntig, wenn die Mandibeln gewaltig stark sind (änsserste Spezialisierung), wie bei Spilopsyllus und den Fellhockern. Die Labialpalpen sind gegliedert. Die Zahl der Glieder variiert. Es scheint, dass eine grössere Zahl Glieder auf primitivere Zustände hinweist: die primitive Macropsylla hat deren 12; obwohl anch spezialisierte Flöhe eine grosse Zahl aufweisen können. Vermipsylla besitzt deren 11-14; dagegen die noch mehr spezialisierten Fellhocker und Spilopsyllus nur 2.

Wagner (in Hor. Soc. Ent. Ross. vol. xxiii. p. 202, 1889) meint, dass zwischen der Dicke der Hant (Epidermis) der Tiere, anf denen der Floh lebt, und der Zahl der Tasterglieder der Unterlippe angenscheinlich eine Beziehung besteht. Als Wagner dies schrieb, kannte er nur eine Art mit vielen Labialpalpengliedern. Jetzt, nun er mehrere Arten kennt, wird er wohl seine Ansicht darüber ändern, denn erstens lebt Macropsylla, mit 12 Labialpalpengliedern, anf Mus velutinus, und zweitens giebt es Suctoria mit sehr langen Mandibeln und Labialpalpen, die ans nicht mehr als 5 Gliedern bestehen, während die Fellhocker, mit gewaltigen Mandibeln, nur zweigliederige Labialpalpen besitzen. Die Gliederzahl ist übrigens sehr gut systematisch zn verwerten, und wie zuerst Rothschild gezeigt hat, anch die Symmetrie oder die Asymmetrie der Labialpalpen.

R. Die Kopfgliederung und Bildung der Fühlergrubendecke.-Es scheint, dass der sehr lange Kopf der Vorfahren der Suctoria sich aus acht Segmenten zusammensetzte, nämlich (1) einem präoralen, (2) einem antennalen, (3) einem labralen 
(4) einem epipharyngealen, (5) einem mandibularen, (6) einem maxillaren, (7) einem hypopharyngealen, und (8) einem labialen. Von diesen Segmenten ist das siebente spurlos verschwunden.

Es scheint, dass die Mundteile sich bald dicht bei einander häuften, dass die frons sich daranf stark entwickelte, und dass daranf;die Antennen weit nach hinten rückten.

Es scheint ferner, dass nachdem dies alles geschehen war, der Kopf-ich habe diese Vermutung auch in der Tijdschrift ausgesprochen-noch sehr lang war, zu lang für die Besitzer, um bequem durch das Haarkleid ihrer Wirte zu schiessen. Es kommt hinzu, dass durch die nach vorn gerichtete Entwickelung der frons der Kopf noch länger wurde als er schon war. Dieses Längerwerden war nicht gerade vorteilhaft für die Schleich- oder Schlupfbewegung dieser Tierchen. Es war darnm vorteilhafter, wenn der Kopf ebenso gegliedert war wie der Thorax und das Abdomen. Und so gliederte der Kopf sich auch wie es scheint in vier Segmente. Diese Glieder sind aber Pseudosegmente, welche nichts mit der ursprünglichen Segmentation zu tun haben. Offenbar sind sie anch entstanden, nachdem die Antennen schon längst weit nach hinten verschoben waren, weit hinter die Mundteile. Denn die hanptsächlichste Gliederung, welche an einigen noch jetzt lebenden Flöhen bestehen geblieben ist, die mittlere, befindet sich hinter den Mundteilen und den Ocellen und vor den Antennen. Wenn man nun fragt, wie diese Gliederungen zu Stande gekommen ist, so können wir nur inbezug auf die mittlere Segmentierung eine Antwort geben.

Hier kamen nämlich die Antennengruben zu Hülfe. An dieser Stelle war der Kopf sehr eingeschnürt, biegsam; die Antennengruben wurden nicht allein tiefer, sondern an dem proximalen Teil derselben formte sich eine Verlängerung derselben, welche endlich in die der Gegenseite überging, sodass der Kopf wirklich "gebrochen" ward.

Aber wie die vorderste Gliederung zu Stande kam, wovon das tuber frontale, der mucro etc., noch die Zengen sind, und anf welche Weise die hinterste entstand, welche zum Teil noch dentlich an Macropsylla und Stephanocircus (Fig. 1 und 2) zu sehen ist, davon habe ich keine Ahnung. Jedenfalls scheint es mir notwendig anzunehmen, dass die Köpfe damals noch nicht gut chitinisiert waren. Es ist undenkbar, dass ein stark ebitinisierter Kopf, anch wenn er lang ist, durch Bengung, oder durch seitlichen Druck, in vier Teile bricht, und dass die auf diese Weise gebrochenen Köpfe erblich geworden sind.

Jede der vier Kopfabteilungen musste, den Lebensgewohnheiten entsprechend, hinten mit einem Kragen (collare) über den nächsten Teil greifen. Vom Kragen des ersten Abschnitts sind nur Spuren übriggeblieben, speziell in einem protectum inversum, welches bei Moeopsylla sjoestedti Rothsch. vorkommt. Die Gliederung zwischen dem zweiten und dritten Abschnitt ist noch bei einigen jetztlebenden Suctoria erhalten. Bei den meisten hat sie aber als solche anfgehört zu existieren. Aber die starken inwendigen chitinösen Ränder sind als falx und als tuber verticale noch anwesend; die Kluft ist als Naht über der falx in vielen Fällen noch angedeutet; der condylus ist als tuber centrale bei einigen Arten erhalten geblieben; ja ich betrachte selbst die chitinöse Lamelle oder Platte, welche als Fühlergrubendecke gedentet werden kann, und welche mehr oder weniger entwickelt bei allen Integricipita vorkommt, als einen unzweifelhaften Rest des collare. Ich bitte den Leser, die Figuren 1 bis 10 miteinander zu vergleichen, und zweifle nicht daran, dass er zugeben wird, dass viel für meine Ansicht spricht. 
Von der dritten Gliederung sind unzweidentige Reste bei Macropsylla und Stephanocircus nachzuweisen, nämlich als tuber postverticale und als falx posterior.

Die Ur-Suctoria mit vier Kopfabschnitten waren offenbar lange Tierchen mit langem Kopfe, nnd mit schlankem, biegsamem, beweglichem Leibe. Sobald sich die Schnelligkeit, womit sie sich zwischen dem Haardickichte hindurcharbeiteten, verminderte, konzentrierten sich die Kopfabteilungen, wurden kürzer. Die zwei vordersten Abteilungen verwuchsen allmählich, und anch die zwei hintersten.

So sind die jetztlebenden Suctoria entstanden. Anf S. 139 gab ich eine Liste der Genera, welche einen in zwei Teile gegliederten Kopf haben. Ich vereinige diese in einem Subordo Fracticipita.

Bei fortwährender Verminderung der Beweglichkeit der Individuen wurde anch die Kopfgliederung überflüssig, sodass anch die zwei übriggebliebenen Abschnitte schon anf die Hälfte ihrer ursprünglichen Länge reduciert und miteinander verwachsen sind, wodurch das caput integrum (also secundär!) entstand. Da nach meiner Meinung alle jetzt lebenden Suctoria, die ein caput integrum haben, miteinander verwandt, gemeinsamen, monophyletischen Ursprungs sind, so vereinige ich sie in einen Subordo Integricipita. Drückt man ein caput integrum zwischen zwei Gläschen, so berstet er immer über den Antennengruben entzwei, wenn über den Antennengruben eine falx oder eine Naht oder selbst nur Spuren davon übrig sind.

Die Spuren der Verwachsung der beiden Kopfabschnitte nahmen von nun an in den zwei Unterabteilungen der Integricipita unabhängig voneinander allmählich ab, sodass wir in diesen Unterabteilungen parallele Reihen zu sehen haben.

S. Die Verbindung des Kopfes mit dem Brustabschnitt.-Der Kopf ist mit seiner ganzen Hinterfläche mit der Vorderfläche des Prothorax verbunden, wie bei den meisten Orthopteren und Coleopteren. Aber statt im Prothorax teilweise eingesenkt zu sein, ist seine Hinterfläche grösser als die Vorderfläche des Pronotnms und greift selbst mit einem breiten Kragen (collare) auf den ersten Brustabschnitt über, ein einzig in der Insektenwelt darstehendes verhalten, welches offenbar wieder in Verbindung mit der Lebensweise steht (nicht direkt die Folge ist) und unbedingt nötig ist für Insekten, welche durch ein Haardickicht dringen.

Wenn nun die Beweglichkeit abnimmt, so wird anch das collare schmäler. Und wenn die Beweglichkeit ganz anfhört, so ist das collare anch nicht mehr nötig, und ist entweder äusserst schmal, oder verschwindet selbst, z. B. in Vermipsylla und den Fellhockern.

T. Die Verkürzung der Thoracalsegmente.-Hand in Hand mit der Verkürzung des ganzen Leibes (siehe oben, S. 143) geht die der Thoracalsegmente, aber in stärkerem Masse. Bei den langen Flöhen ist die Länge des Kopfes und des Thorax zusammengenommen oft grösser als die des Abdomens. Bei kürzeren Flöhen, z. B. Pulex, ist der Thorax nicht länger als der etwas grosse Kopf. Bei den sich gar nicht mehr bewegenden "Sarcopsyllidae" ist er sogar kürzer als das erste Abdominaltergit. Bei Echidnophaga ist das Metanotum am Rücken fast unterdrückt, bei Dermatophilus dort selbst mit dem Mesonotum verwachsen.

$U$. Die Verbindung der Thoracal- und Abdominalsegmente.-Selbstrerständlich besitzen alle Thoracalsegmente denselben Kragen wie die Kopfabschnitte. Auch diese Erscheinung ist einzig unter den Insekten. Aber die übergreifenden Rander der Abdominalabschnitte finden sich auch lei anderen schnell sich bewegenden Incekten. Dass diese Kragen in Verbindung stehen mit der schnellen Bewegung 
zwischen Haaren, beweisst anch das fast vollkommene Fehlen derselben bei den Fellhockern, bei denen anch kein Grund für ihre Existenz mehr vorhanden ist.

V. Die Zahl der Antepygidialborsten.-Nach meiner Meinung sind Antepygidialborsten spezialisierte Borsten. Letztere stehen in Querreihen, welche eine ziemlich grosse Zahl Borsten enthalten. Mithin ist der Besitz von mehreren Antepygidialborsten, wie bei Nycteridopsylla und Ctenophthalmus, ein primitiveres Merkmal als die Anwesenheit von nur einer dieser Borsten, wie bei den meisten Flöhen. Und die Abwesenheit dieser Borsten, wie bei Vermipsylla und Chaetopsylla, bedentet starke Spezialisierung.

W. Die An- und Abwesenheit der cerci.-Nach den Untersuchungen von Lass haben sich die cerci der Weibchen wahrscheinlich ans den Nachschiebern der Larve entwickelt. Ist dies der Fall, dann sind die cerei primitive Organe nnd ist die Abwesenheit derselben ein secundäres Merkmal, ein phylogenetisch junger Character. Dieser Gedankengang wird durch die Tatsache bestätigt, dass fast alle Flöhe cerci besitzen und die Abwesenheit derselben nur bei spezialisierten Formen vorkommt.

X. Das doppelte Receptaculum seminis.-Wagner (in Zool. Anz. vol. xxi. p. 148, 1903) hat zuerst nachgewiesen, dass Hystrichopsylla ein doppeltes Receptaculum seminis besitzt, oder besser: zwei Receptacula. In 1905 (in Nov. Zool. vol. xii. p. 486) beschrieb Rothschild eine zweite Gattung, Macropsylla, welche ebenfalls zwei Receptacula seminis hat. Derselbe Autor meldete 1908 (in Parasitology vol. i. pp. 5 und 91) eine dritte Gattung, Coptopsylla, mit zwei Receptacula. Ich selbst publicierte einen vierten Fall (in Ent. Berichten vol. ii., 1909). Ich fand nämlich anch bei Typhloceras zwei Receptacnla seminis.

Da die drei erstgenannten Flöhe primitive Formen sind, so muss man wohl annehmen, dass der Besitz von zwei Receptacula seminis auf primitive Verhältnisse hinweist. Typhloceras, obwohl selbst keine so primitive Form, hat also doch in ihren zwei Receptacnla etwas Primitives bewahrt.

Y. Allgemeine Betrachtungen.-Die Gruppe der Suctoria scheint mir eine durch Parasitismns in Degenerierung begriffene, sehr primitive Gruppe zu sein. Die Länge des Kopfes und der Besitz von zwei Receptacula seminis, welche keine andere Insektenordnung anfweisen kann, deuten auf eine uralte Abzweignng von dem Insektenstamme. Mit welchen anderen Insekten sie verwandt sind, scheint immer rätselhafter zu werden. Das Durchmachen einer Metamorphose, oder eines Larvenstudiums, selbst eines, das denen der Diptera gleicht, braucht noch keine Verwandtschaft mit den letztern anzudenten; denn die secundär erworbene Larve, als Folge einer besonderen Lebensweise der jungen Flöhe, kann bei den Suctoria nnabhängig von den anderen Insektenordnungen entstanden sein. Auch das Puppenstadium ist kein Beweis von Verwandtschaft. Es kann ebenfalls unabhängig von den anderen Insekten in den Lebenscyclus der Suctoria eingeschaltet sein. Kommt ja doch ein Puppenstadium auch bei den Männchen der Cocciden, einer Abteilung der Rhynchoten, vor, bei denen ein Puppenstadium etwas ganz Fremdes ist!

\section{Systematischer Teil.}

Eine grosse Anzahl der jetztlebenden Suctoria zeigen ncch die schlanke Gestalt und den gliederten Kopf der Ur-Suctoria, also sehr primitive Merkmale. Ihre Antennen sind zwölfgliederig ; die der Männchen sind lang, überragen in der Ruhe oft den Kopfrand, sodass der distale Teil in eine in den Propleuren eingesenkte Grube zu liegen kommt, oder, wie es mehrfach ausgedrückt worden, die 
Antennengrube sich auf die Propleuren fortsetzt. Alle diese Formen bilden, meines Erachtens, einen einheitlichen primitiven Subordo, welchen ich Fracticipita (in Tijd. v. Ent. vol. li. p. 92 ; 6 Mai 1908, significatio emendata) genannt habe. Hierzn gehören die auf S. 139 genannten Gattungen.

Unter diesen bilden Macropsylla und Stephanocircus gewiss eine ältere Gruppe, gekennzeichnet durch ihren tuber postverticale. Daher nenne ich sie Posttuberata (nova Superfamilia). Sie steht den anderen jüngeren Fracticipita gegenüber, welche gar keinen tuber mehr aufweisen, und die zusammen die nova Superfamilia der Intuberata bilden.

Alle anderen Gattungen gehören zu dem Subordo der Integricipita (ebenda : significatio emendata), die ein caput integrum, einen Kopf aus einem Stück, besitzen, welcher aber ein ganz anderes caput integrum ist, als es andere Insekten haben, denn er ist, wie ich oben betont habe, secundär entstanden. Der monophyletische Ursprung dieser Gruppe ist, obwohl nicht unanfechtbar, doch sehr wahrscheinlich. Sie schliesst sich jedenfalls den Fracticipita an, und zwar wahrscheinlich durch die Gattungen Neopsylla und Spalacopsylla an die Gattung Palaeopsylla der Fracticipita.

Unter den Integricipita besitzen viele Gattungen eine lange, ovale, oder elliptische Fühlerkenle (Fig. 13, 14, 19). Sie bilden offenbar eine einheitliche Gruppe, welche ich Superfamilia Longiclavata nennen will.

Die übrigen Gattungen haben Antennen mit kurzer, runder clava (Fig. 15-18). Die clava zeigt noch eine andere Besonderheit; das freie Ende des ersten Gliedes ist mehr oder weniger mandolinenförmig. Diese Gattungen sind offenbar miteinander verwandt, und bilden Superfamilia Breviclavata.

Welche Gattung der Breviclavata am nächsten mit irgend einer Gattung der Longiclavata verwandt ist, kann ich nicht entscheiden. Hoffen wir, dass noch einmal Übergangsformen gefunden werden. Ich sehe keine Verwandtschaft zwischen Pulex und Ctenocephalus einerseits und Spilopsyllus anderseits.

Sowohl bei den Longiclarata als bei den Breviclarata finden wir Formen, die durch die Gewohnheit sich festzusaugen, Fellhocker geworden sind, das heisst, äusserst kurze Flöhe mit einer Fühlerkeule, in der die meisten Glieder miteinander rerwachsen sind, mit äusserst kurzem Thorax, und mit sehr ansdehnbarem Abdomen der Weibchen.

Die langen Formen unter den Longiclavata, mit langem Thorax, vereinige ich als Dolichothoracica (nova Sectio); während zu den Brachythoracica (nova Sectio) nur die Fellhocker, mit kurzem Thorax, gehören. Diese schliessen sich meines Erachtens am besten an Spilopsyllus an.

Die Superfamilia der Breviclavata wird ebenfalls eingeteilt in lange Formen, mit langem Thorax, und in kurze Formen, mit kurzem Thorax, oder in die Solitothoracica (in Entom. Bericht. vol. ii. p. 252, Juli 1908, significatio emendata) und Brevithoracica (ebenda: significatio emendata). Wie diese sich anschliessen an die Solitothoracica ist nicht dentlich; vielleicht an Pulex-ähnliche Formen. Nene Funde bringen hoffentlich mehr Licht.

Selbstverständlich sind mit dieser neuen Einteilung einige Familien anfgehoben; andere Familien habe ich vereinigen müssen; für neue Familien wählte ich neue Namen. So viel wie möglich behielt ich schon bekannte Namen bei, obwohl oft mit " significatio emendata."

Ich gebe hier eine Übersicht, welche zugleich als Bestimmungstabelle dienen kann, 


\section{Klassification der Suctoria.}

A. Lange Flöhe, mit gegliedertem Kopfe, mit Ctenidien sowohl am Kopfe als am Thorax, mit lang-ovaler, freigliederiger clava. Subordo Fracticipita Oudemans 1908.

B. In der pars posterior des Kopfes ist noch dentlich eine Spur einer früheren Gliederung dieses Abschnittes übriggeblieben und zwar in der Form eines tuber postverticale mit falx posterior. Kein Auge. Superfamilia Posttuberata Oudemans 1909 (nova). Entbält nur eine Familie: Macropsyllidae Oudemans 1909 (nova).

C. Subfrontale, genale und anteantennale Ctenidien bilden keinen Helm. Macropsylla Rothsch. 1905.

CC. Subfrontale und anteantennale Ctenidien bilden einen Helm. Stephanocircus Skuse 1890.

BB. In der pars posterior des Kopfes ist keine Spur eines tuber postverticale mehr wahrzunehmen. Superfamilia Intuberata Ondemans 1909 (nova).

C. Mit genalen oder anteantennalen Ctenidien. Maxillen spitz. Labialpalpen 5-gliederig, symmetrisch. Auge scilecht entwickelt oder abwesend. Familia Hystrichopsyllidae (-nae) Tiraboschi 1904 (-dae Baker 1905). (Trichopsyllidae Tiraboschi 1904), (Ctenopsyllidae Baker 1905).

D. Nur Protibia distal mit geschlossener Borstenreihe. Hystrichopsylla Tasch. 1880.

DD. Alle Tibien mit geschlossener Borstenreihe. Ctenophthalmus Kolen. 1856.

DDD. Tibien nur mit Kerben und Doppelborsten. Kopf mit tuber frontale, mit oder ohne mucro. Palaeopsylla Wagner 1903.

CC. Nur $(2 x) 2$ subfrontale Ctenidien. Maxillen stumpf bis spitz. Labialpalpen 5-gliederig, symmetrisch. Angen rudimentär oder abwesend. Familia Ischnopsyllidae Wahlgren 190\%. (Typhlopsyllinae Tiraboschi 1904). (Ceratopsyllidae Baker 1905).

D. Pro- und Mesotibien ganz, Metatibia nur distal mit geschlossener Borstenreihe. Maxillen spitz. Thaumapsylla Rothsch. 1907.

DD. Alle Tibien mit Kerben und Doppelborsten. Maxillen mehr oder weniger stumpf.

E. Vielt Antepygidialborsten, einem Ctenidium ähnlich. Nycteridopsylla Oudemans 1906.

EE. Nur eine Antepygidialborste.

F. Metepimerum ohne Ctenidium. Ischnopsyllus Westwood 1833.

FF. Metepimerum mit Ctenidium. Chiropteropsylla Oudemans 1908.

CCC. Mit genalem Ctenidium. Maxillen spitz. Labialpalpen asymmetrisch (hinten häntig). Ange anwesend. Familia Typhloceratidae Ondemans 1909 (nova).

AA. Kopf ungegliedert, d. h. ans zwei oder mebr Gliedern verwachsen, wovon oft noch Spuren vorhanden sind und zwar in der Form eines tuber verticale, einer falx, einer Naht. Subordo Integricipita Ondemans 1908.

B Clava lang, oval. Superfamilia Longiclavata Ondemans 1909 (nova). 
C. Flöhe fast immer lang. Clava freigliederig. Thorax nicht kürzer als der Kopf, länger als das erste Tergit. Sectio Dolichothoracica Ondemans 1909 (nova).

D. Kopf (Gena) und Pronotum mit Ctenidium. Familia Neopsyllidae Oudemans 1909 (nova).

E. Labialpalpen 4-oder 5-gliederig, symmetrisch. Subfamilia Neopsyllinae Oudemans 1909 (nova).

F. Mit tnber verticale, schwerer falx, tuber frontale, mucro oder listron. Labialpalpen 5-gliederig. Neopsylla Wagner 1903.

FF. Mit leichter falx. Labialpalpen 5-gliederig. Spalacopsylla Ondemans 1906.

FFF. Keine Spur von falx. Tuber frontale mit Grube, worin listron oder protectum. Mit Ange. Listropsylla Rothsch. 1907.

EE. Labialpalpen 2-gliederig, durchsichtig, häntig. Subfamilia Spilopsyllinae Oudemans 1909 (nova). Mit nur einem Genus : Spilopsyllus Baker 1905.

DD. Kopf ohne, Pronotum mit Ctenidium. Familia Dolichopsyllidae Baker 1905 .

E. Labialpalpen 5-gliederig, symmetrisch. Subfamilia Dolichopsyllinae Baker 1905.

F. Mit falx. Mit protectum. Mit Ange. Innenseite der Metacoxa mit Dörnchen. Odontopsyllus Baker 1905.

FF. Mit falx. Mit protectum. Mit Ange. Innenseite der Metacoxa ohne Dörnchen. Dasypsyllus Baker 1905.

FFF. Mit schwacher falx. Ohne protectum. Mit Ange. Pygiopsylla Rothsch. 1906.

FFFF. Mit weisser Naht. Mit protectum. Ohne Auge. Dolichopsyllus Baker 1905.

FFFFF. Nur mit protectum und Ange. Ceratophyllus Curtis 1829 .

EE. Labialpalpen 4- oder 5-gliederig, asymmetrisch (hinten häntig), distal spitz. Subfamilia Hoplopsyllinae Ondemans 1909 (nova). Mit einem Genus : Hoplopsyllus Baker 1905.

DDD. Kopf und Pronotum ohne Ctenidium. Familia Anomiopsyllidae Oudemans 1909 (nova).

E. Labialpalpen 5-oder 6-gliederig, symmetrisch. Subfamilia Anomiopsyllinae Baker 1905. (Lycopsyllidae Baker 1905 ; Malacopsyllidae Baker 1905 ; Megapsyllidae Baker 1898).

F. Mit starker falx. Mit tuber frontale und protectum inversum. Mit Auge. Parapsyllus Enderl. 1903.

FF. Mit starker falx. Mit Auge. Malacopsylla Weyenb. 1881.

FFF. Mit Spur eines tuber verticale. Mit Naht. Mit Auge. Goniopsyllus Baker 1905.

FFFF. Mit schwacher Spur von falx (branner Anflug über der Fühlergrube). Mit mucro oder protectum hoch am Kopfe. Lycopsylla Rothsch. 1904.

FFFFF. Mit tuber frontale. Mit Ange. Chaetopsylla Kohant 1903. 
FFFFFF. Mit Auge. Gena mit kurzem, breitem, stumpfem Anhang. Coptopsylla Jordan und Rothsch. 1908.

FFFFFFF. Kein Ange. Wenig Behaarung. Anomiopsyllus Baker 1904.

EE. Labialpalpen \%- bis 14-gliederig, symmetrisch. Subfamilia Vermipsyllinae Wagner 1889 (-da Wagner; -dae Baker 1905; -nae Baker 1905). Mit einem Genus: Vermipsylla Schimk. 1885 .

CC. Flöhe sehr kurz. Clava verwachsengliederig. Thorax viel kürzer als der Kopf und als das erste Tergit. Sectio Brachythoracica Oudemans 1909 (nova). Mit nur einer Familia: Hectopsyllidae Baker 1904. (Sarcopsyllidae Tasch. 1880; Rhynchoprionidae Baker 1906 ; Hectoropsyllidae Oudemans 1906 ; Dermatoplilidae Ondemans 1906.)

D. Clava verwachsen 8-gliederig. Maxillen stumpf. Hectopsylla Franenf. 1860.

DD. Clava verwachsen 7-gliederig. Maxillen spitz. Rhynchopsylla Haller 1880.

DDD. Clava verwachsen 6-gliederig. Maxillen stumpf. Frons mit hufeisenförmiger Rinne über dem nasenförmigen Vorsprung. Dermatoplilus Guérin 1838.

BB. Clava kurz, rund ; freier Teil des ersten Gliedes der Clava mandolinenförmig. Flöhe gedrungen. Superfamilia Breviclavata Oudemans 1909 (nova).

C. Thorax nicht kürzer als der Kopf, länger als das erste Tergit. Clava frei- oder teilweise verwachsengliederig. Sectio Solitothoracica Oudemans 1908.

D. Kopf und Pronotum mit Ctenidium. Labialpalpen asymmetrisch (immer?). Familia Archaeopsyllidae Oudemans 1909 (nova).

E. Mit subfrontalem (4), genalem (4), und angularem (1) Ctenidium. Ctenocephalus Kolen. 1857.

EE. Mit genalem (2-3) und angularen (1) Ctenidium. Archaeopsylla Dampf 1908.

DD. Kopf ohne, Pronotum mit Ctenidium. Labialpalpen symmetrisch (immer?). Familia Uropsyllidae Oudemans 1909 (nova). Mit Genus Uropsylla Rothsch. 1905.

DDD. Kopf und Pronotum ohne Ctenidium. Familia Pulicidae Tasch. 1880.

E. Labialpalpen symmetrisch. Subfamilia Rhopalopsyllinae Oudemans 1909 (nova). Mit Genus Rhopalopsyllus Baker 1905.

EE. Labialpalpen asymmetrisch (hinten häntig). Subfamilia Pulicinae Tirab. 1904.

F. Mesosternit schmal, ohne inwendige stabförmiger Chitinisation von der Insertion der Coxa anfwärts. Pulex L. 1758.

FF. Mesosternit breit, mit inwendiger stabförmige Chitisination von der Insertion der Coxa aufwärts.

G. Mit falx. Mit protectum inversum. Gena mit dreieckigem Läppchen wie bei den Hectopsyllidae. Mit Auge. Moeopsylla Rothsch. 1908. 


\section{( 158$)$}

GG. Mit dentlicher Spur von falx. Mit Ange. Ċlava freigliederig. Ornithopsylla Rothsch. 1908.

GGG. Mit Andentung einer falx. Mit Ange. Gena mit dreieckigem, scharfem Anhang. Pariodontis Jord, und Rothsch. 1908.

GGGG. Nur mit Ange. Loemopsylla Jord, und Rothsch. 1908. CC. Thorax viel kürzer als der Kopf uud als das erste Tergit. Sectio Brevithoracica Ondemans 1908. Mit nur einer Familie : Echidnophagidae Oudemans 1909 (nova). Mit nur einer Gattung : Echidnophaga Olliff 1886.

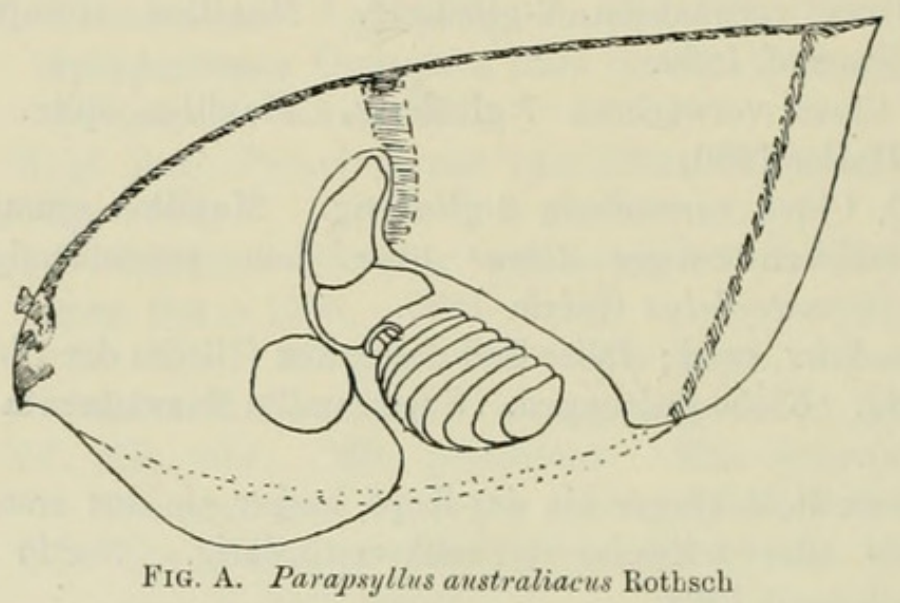




\section{$2 \mathrm{BHL}$ Biodiversity Heritage Library}

Oudemans, A. C. 1909. "Neue Ansichten über die Morphologie des Flohkopfes, sowie über die Ontogenie, Phylogenie und Systematik der Flöhe." Novitates zoologicae : a journal of zoology in connection with the Tring Museum 16, 133-158. https://doi.org/10.5962/bhl.part.21962.

View This Item Online: https://www.biodiversitylibrary.org/item/21989

DOI: https://doi.org/10.5962/bhl.part.21962

Permalink: https://www.biodiversitylibrary.org/partpdf/21962

\section{Holding Institution}

Natural History Museum Library, London

\section{Sponsored by}

Natural History Museum Library, London

\section{Copyright \& Reuse}

Copyright Status: Public domain. The BHL considers that this work is no longer under copyright protection.

This document was created from content at the Biodiversity Heritage Library, the world's largest open access digital library for biodiversity literature and archives. Visit BHL at https://www.biodiversitylibrary.org. 